\title{
Research Paper \\ The Effects of Cognitive-metacognitive Strategies Training and Positive Psychology \\ Training on Academic Self-efficacy, Academic Burnout, and Learning Strategies
}

${ }^{*}$ Mohammad Abbasi ${ }^{1}$, Hossein Davoodi ${ }^{1}$, Hassan Heidari ${ }^{1}$, Zabih Pirani ${ }^{2}$

1. Department of Psychology, Islamic Azad University, Khomein Branch, Khomein, Iran

2. Department of Psychology, Faculty of Humanities, Islamic Azad University, Arak Branch, Arak, Iran.

\begin{tabular}{|l|l|l|l}
\hline $\begin{array}{l}\text { Use your devic to scan } \\
\text { and read thearticle online }\end{array}$ & $\begin{array}{l}\text { Cftation: Abbasi, M., Davoodi, H., Heidari, H., \& Pirani, Z. (2021). [The Effects of Cognitive-metacognitive Strategies Train- } \\
\text { ing and Positive Psychology Training on Academic Self-efficacy, Academic Burnout, and Learning Strategies (Persian)]. Journal } \\
\text { of School Psychologh and Institutions, 9(4):106-119. https://doi.org/10.32598/JSPI.9.4.9 }\end{array}$ \\
dol'https://doi.org/10.32598/JSPI.9.4.9
\end{tabular}

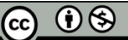

Article Info:

Received: 11 Sep 2020

Accepted: 09 Jan 2021

Available Online: 01 Jan 2021

Key words:

Academic burnout, Academic self-efficacy, Cognitive-metacognitive, Learning strategies, Positive psychology

\section{A B S T RACT}

Objective The present research aimed to explore the effects of cognitive-metacognitive strategies and positive psychology training skills on academic self-efficacy, academic burnout, and learning strategies. Methods This was a quasi-experimental with an unequal control group design. The statistical population included students in Arak City, Iran, in the academic year 2019-2020. Accordingly, 45 of whom were selected using the convenience sampling method and randomly divided into 3 experimental and control groups ( $n=15 / g r o u p)$. The study participants completed the Academic Self-Efficacy Scale, Bresso et al.'s (1997) Burnout Questionnaire, and the Learning Strategies Scale. The study hypotheses were tested by Multivariate Analysis of Covariance (MANCOVA) and One-way Analysis of Variance (ANOVA).

Results The present study data indicated that concerning self-efficacy and academic burnout, teaching cognitive-metacognitive strategies was more effective than positive psychology training $(\mathrm{P}<0.01)$.

Conclusion The present research results that teaching cognitive-metacognitive strategies and positive psychology promoted academic self-efficacy and learning strategies and reduced academic burnout.

\section{Extended Abstract}

\section{Introduction}

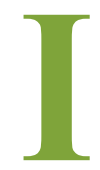

dentifying the factors affecting the academic achievement and performance of learners, creates an appropriate approach to planning and development as well as the evolution of educational programs. It can be used to achieve the most optimal results for the educational institution as well as the learners (Halpany et al., 2010). A critical characteristic affecting academic performance is self-efficacy beliefs. Academic self-efficacy is an individual's judgment of their ability to perform a particular activity, overcoming obstacles to achieving goals in a given situation (Narimani, Khoshnodnia Chamachaei, \& Ghasemi, 2013). Academic self-efficacy refers to a subject's belief in how well they can complete homework at designated levels. The results of research by Bargi et al. (2019), Versava and Fuglava (2018), Veda et al. (2018), Nakhostin Goldost et al. (2019), and Ghasemi et al. (2018) highlighted those teaching learning strategies impacted academic self-efficacy. Another influential component in the field of education is academic burnout. Academic burnout refers to feeling tired because of the de-

\section{* Corresponding Author:}

Mohammad Abbasi

Address: Department of Psychology, Islamic Azad University, Khomein Branch, Khomein, Iran.

Tel: +98 (918) 4321553

E-mail: asmani200090@yahoo.com 
mands and requirements of education (emotional fatigue), having a pessimistic and disinterested in homework (disinterest or pessimism), and feeling unworthy as a learner (low efficiency or inefficient) (Shaofli et al., Quoting Hayati et al., 2012). An effective component in education is the application of learning strategies by learners. Learning is the process of making relatively lasting changes, i.e., the result of experiences and cannot be attributed to temporary states of the body and what results from illness, fatigue, or medications (Kimble 1991, Quoted by Seif, 2018). Cognitive and metacognitive strategies are measures that help in learning and remembering. Cognition and metacognition include learning, remembrance, and thinking; thus, these strategies are also methods of learning and thinking (Seif, 2018). Given the impact of cognitive-metacognitive strategies on the learning process as well as the focus of positive psychology on individual abilities and talents and the use of capabilities in the learning process, the effectiveness of cognitive-metacognitive strategies training and the difference between self-efficacy, academic burnout, and learning strategies should be tested.

\section{Materials and Methods}

This was an applied and quasi-experimental research with an unequal control group design. The study population included all high school students in Arak City, Iran, in the academic year of 2019-2020. Of them, 45 subjects were selected by convenience sampling method and randomly assigned to groups of 15 individuals. In terms of gender, $66.7 \%$ of the study participants were girls and $33.3 \%$ were boys. Moreover, $8.8 \%$ of the research sample had a grade point average between 14 and $16 ; 62.2 \%$ had a grade point average between 16 and $18 ; 29 \%$ had a grade point average between 18 and 20 .

\section{Results}

Table 4 indicates that the calculated F-value was significant. In other words, cognitive-metacognitive strategies and positive psychology training were effective on at least one of the dependent variables. The Eta coefficient suggested that the intensity of the effectiveness of education equaled $90 \%$. The test power revealed that if this training is performed 100 times, these results will be obtained in every 100 cases. Furthermore, the test power of one reflects the adequacy of the sample size. Table 5 highlights that the calculated F-value was significant for academic self-efficacy, academic burnout, and learning strategies. In other words, the provided training significantly impacted all research variables. The Eta coefficient suggested that the highest effect concerned academic self-efficacy and the least effect respected academic burnout. As per Table 6, there was a significant difference in all research variables between the control group and the experimental groups of cognitivemetacognitive strategies training and positive psychology training. In terms of academic self-efficacy, teaching cognitive-metacognitive strategies was more effective than teaching positive psychology. Concerning academic burnout, teaching cognitive-metacognitive strategies and teaching positive psychology, respectively were influential. Moreover, concerning learning strategies, teaching cognitive-metacognitive strategies were more efficient than teaching positive psychology.

\section{Discussion and Conclusion}

The present study compared the effects of cognitivemetacognitive strategies training and positive psychology training on academic self-efficacy, academic burnout, and learning strategies. The obtained results signified a difference between the effects of teaching cognitive-metacognitive strategies and positive psychology training on academic self-efficacy. In the selfefficacy variable, teaching cognitive-metacognitive strategies, followed by teaching psychological psychology were effective. These results were consistent with those of Goldust et al. (2019), Ghasemi and Yousefian Amirkhiz (2018), Bargi et al. (2019), Versava and Foglava (2018), Veda et al. (2018), as well as Anwar and associates (2018).

\section{Ethical Considerations}

\section{Compliance with ethical guidelines}

All ethical principles are considered in this article. The participants were informed about the purpose of the research and its implementation stages. They were also assured about the confidentiality of their information and were free to leave the study whenever they wished, and if desired, the research results would be available to them.

\section{Funding}

This research did not receive any grant from funding agencies in the public, commercial, or non-profit sectors.

\section{Authors' contributions}

All authors equally contributed to preparing this article.

\section{Conflicts of interest}

The authors declared no conflict of interest. 
This Page Intentionally Left Blank 
سنجش اثربخشى آموزش راهبردهاي شناختى فراشناختى و آموزش روانشناسى مثشتئَ بر

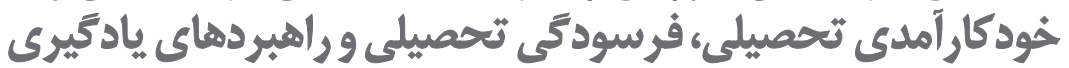

"محمد عباسى'، حسين داودى'، حسن حيدرى'، ذييح ييرانى'

ا. ب. كروه روانشناسى، دائشكاه آزاد اسلامى، واحد خمين، خمين، ايران.

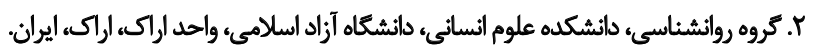

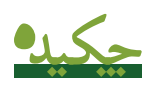

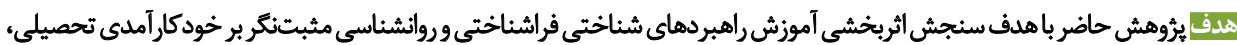

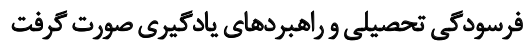

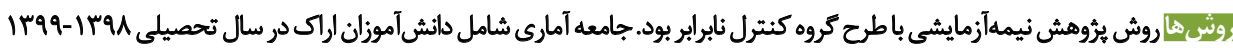

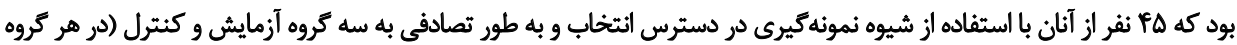

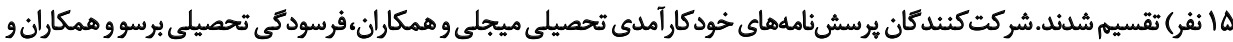

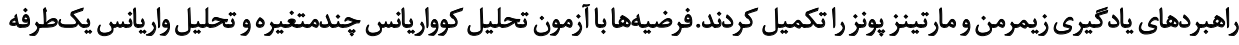

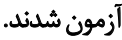

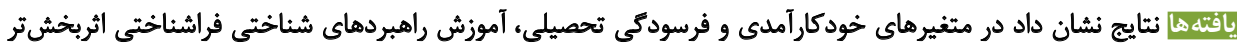

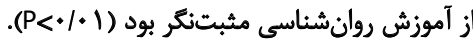

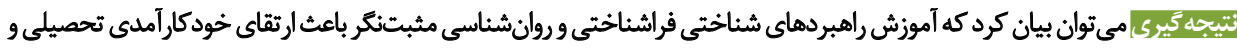

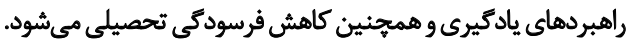

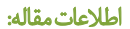

تاريخ دريافت: آب شهريور

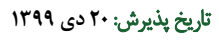

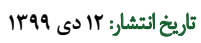

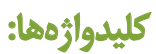

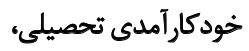
راهبردهاى شناختى تركيلى

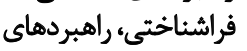
يادكيرى، روانشناسى فئرى

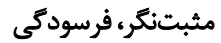
تحصيلى
\end{abstract}

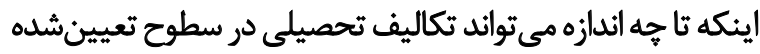

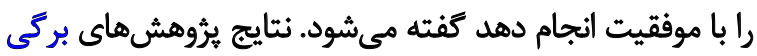

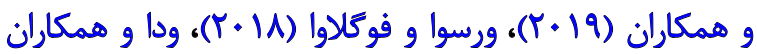

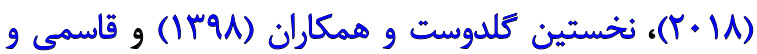

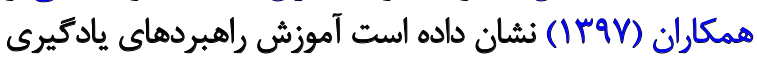
بر خودكارآمدى تحصيلى تأثير دارد.

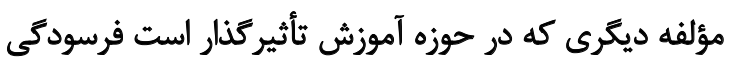

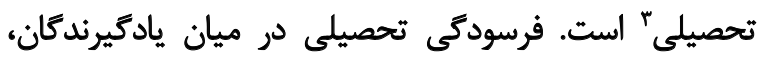

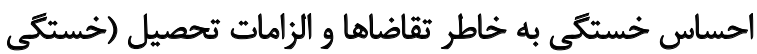

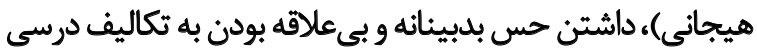

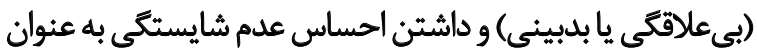
يك يادكيرنده (با كارآمدى بايين يا فقدان كارايى) اشاره دارد

شناسايى عوامل تأثيركذار بر بيشرفت و وملكريكرد تحصيلى برئي

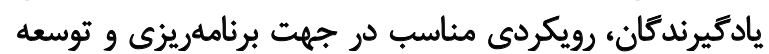

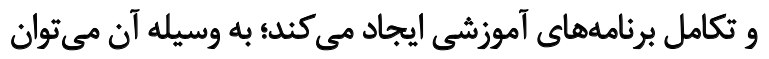

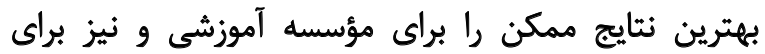

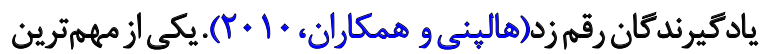

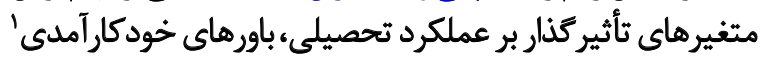

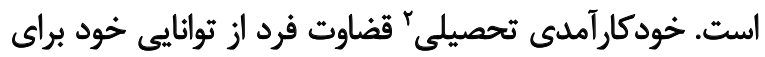

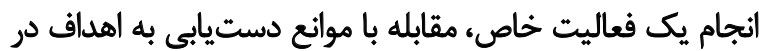

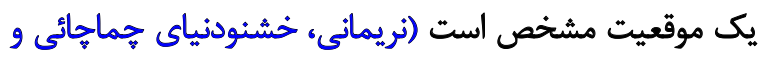

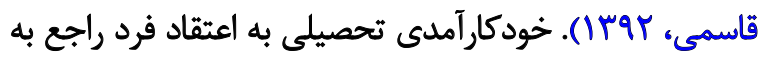

\section{Self-efficacy}


شناختى و هدايت آنها هستند. راهبردهاى فراشناختى عمده

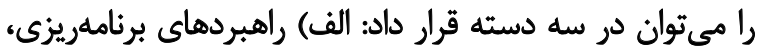

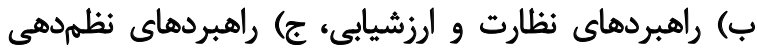

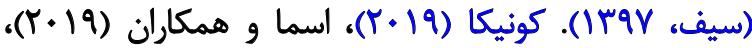

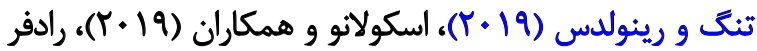

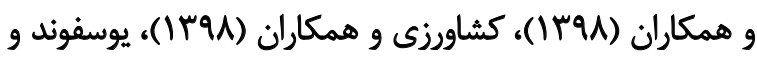

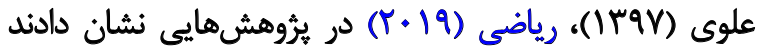

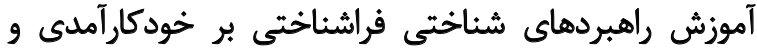

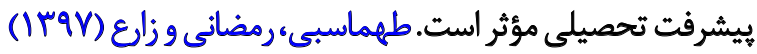

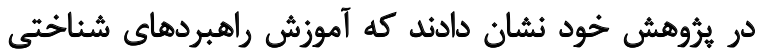
فراشناختى موجب كاهش فرسودمى تحصيلى مى شود. روشهاى متنوعى براى افزايش خودكارآمدى تحصيلى و وادئل

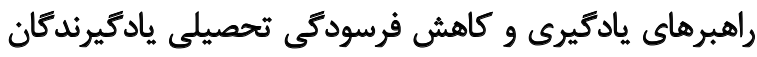

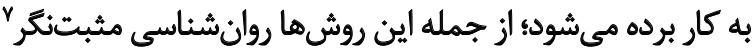

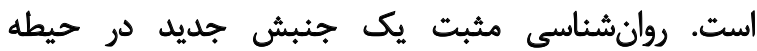

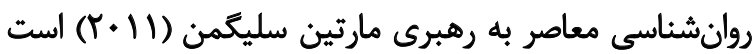

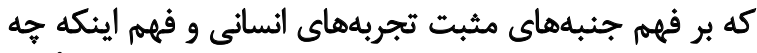

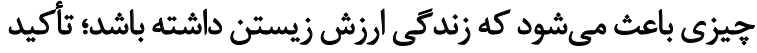

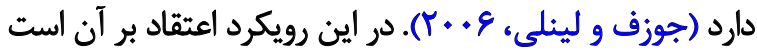

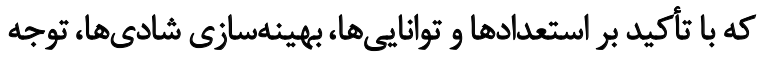

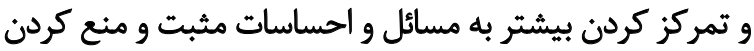

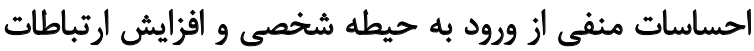

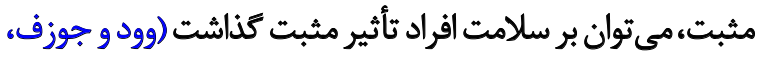

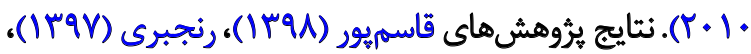

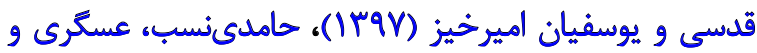

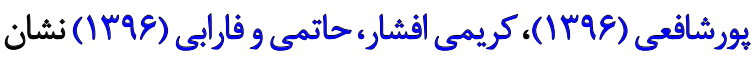

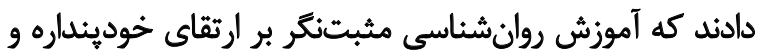
خودكارآمدى دانش آموزان اثر مثبت دارد.

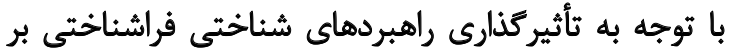

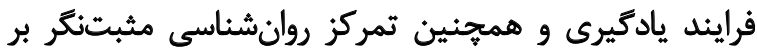

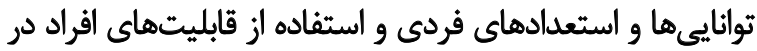

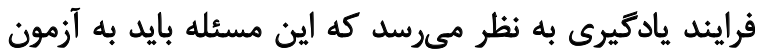

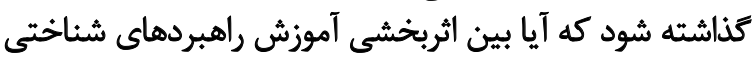

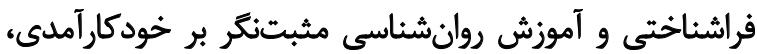
فرسودىى تحصيلى و راهبردهاى يادئيرى تفاوت وجود دارده؟

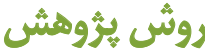

اين يُوهش از نوع كاربردى با روش نيمهآزمايشى از نوع طرح

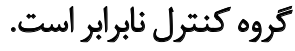

جامعه، نمونه و روش نمونه كيرى: دانشآموزان دوره متوسطه

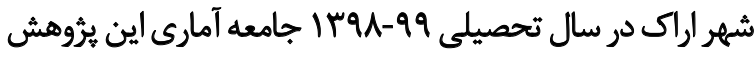

7. Positive psychology

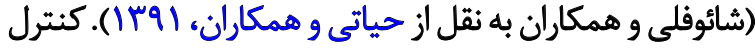

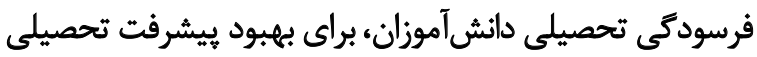

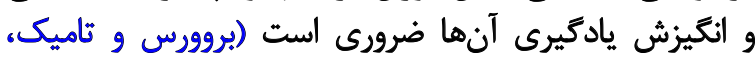

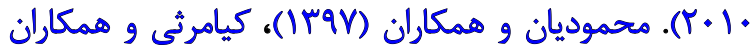

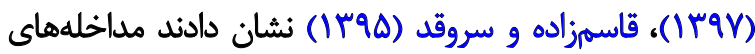
روانشناسى مثبتنكر و اشتياق عاطفى، شناختى و و رفتارى

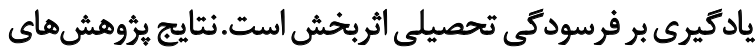

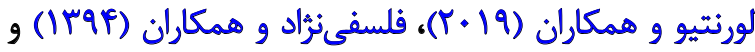

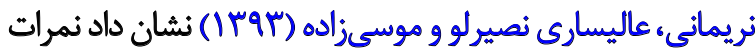

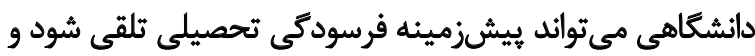
خودكارآمدى بر فرسودىى تحصيلى تأثير معنادارى داردي.

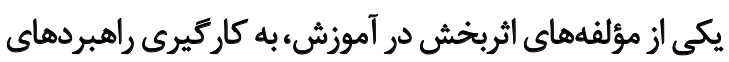

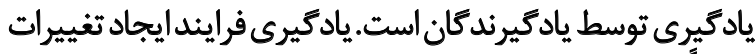

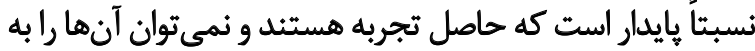

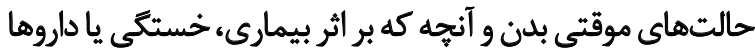

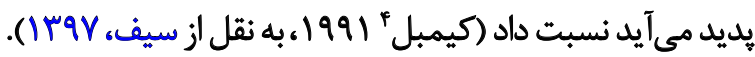

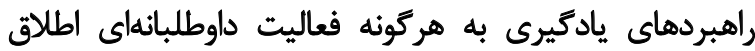

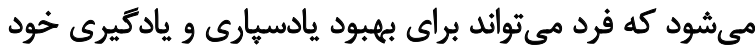

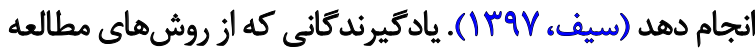

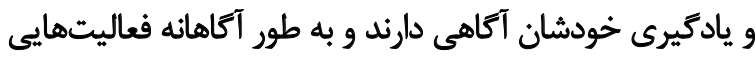

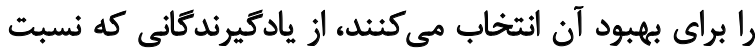

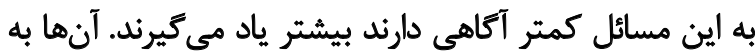

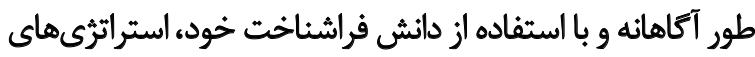

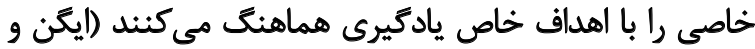

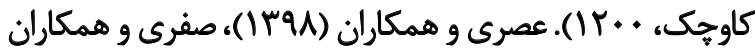

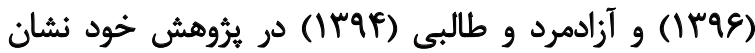

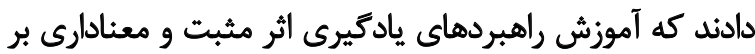

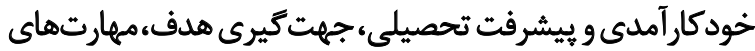
تحليل، خلاقيت و عمل دارد.

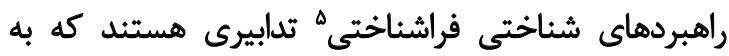

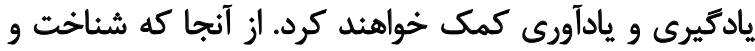

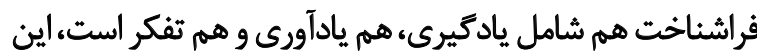

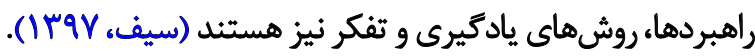

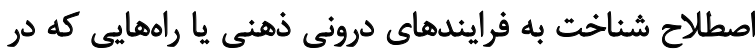

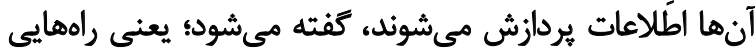

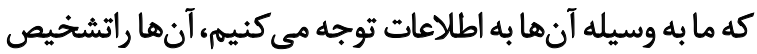

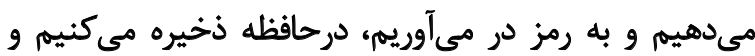

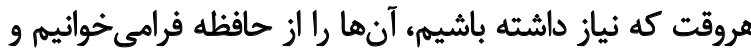

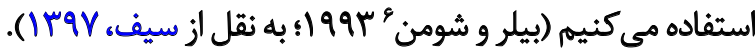
راهبردهاى فراشناختى تدابيرى براى نظارت بر راهبردهاى

\section{Kimble}

5. Cognitive \& Meta Cognitive strategies

6. Bilere \& Showman 


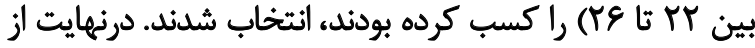

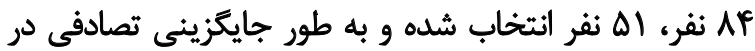

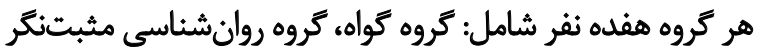

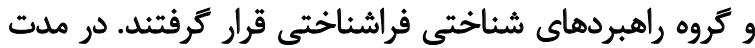

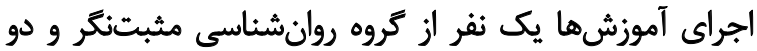

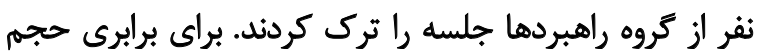

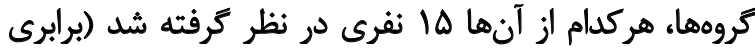

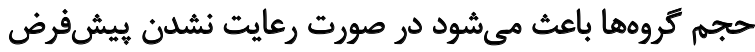

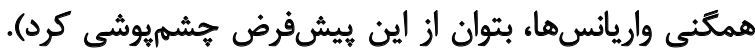

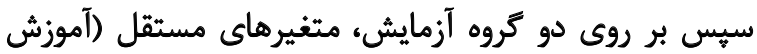

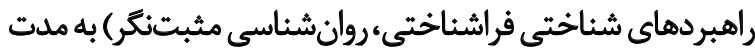

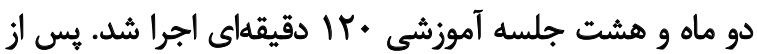

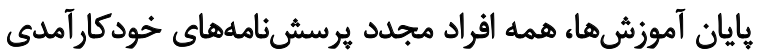

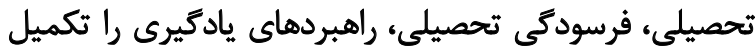

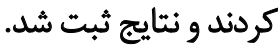

سرفصل جلسات أموزش راهبردهاى شناختى فراشناختى

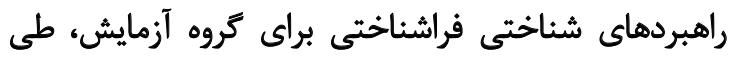

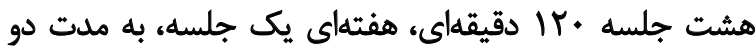

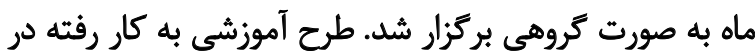

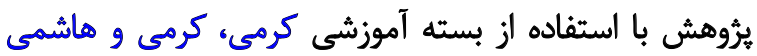

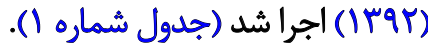
سرفصل جلسات أموزشى روانشناسى مثبتثكّر سرفصل جلسات آموزشى روانشناسى مثبتنكر بر اساس

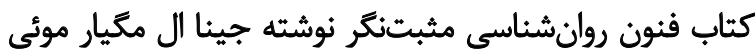

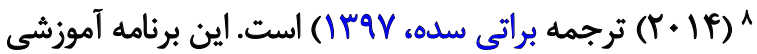

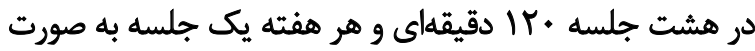

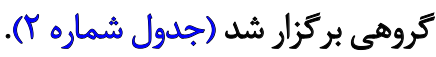

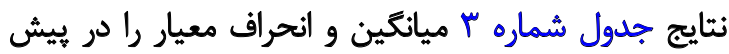

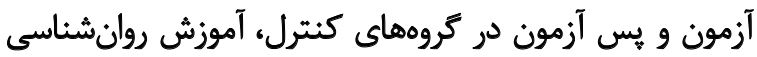

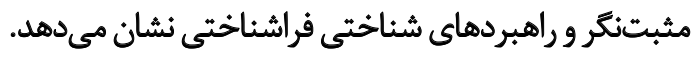
به منظور محاسبه تحليل كوواريانس جندمتغيرى ابتدا بيش

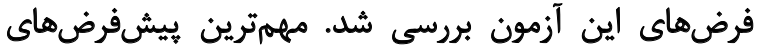

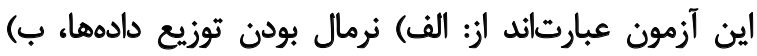

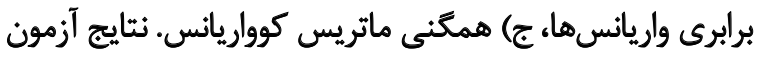

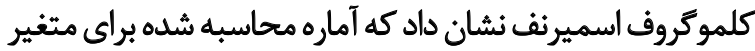

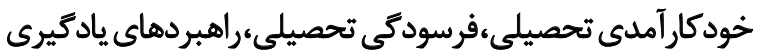

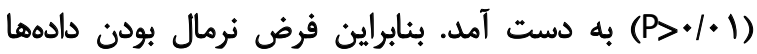

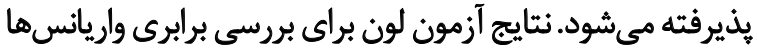

8. Gina El Magyar - Moie
را تشكيل دادند. از جامعه آمارى يرّوهش حاضر fQ أنفر به روش

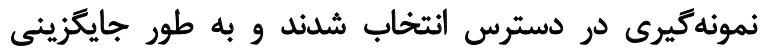

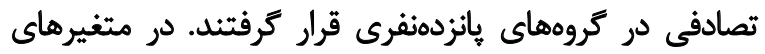

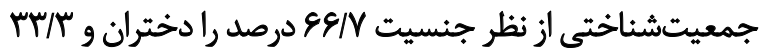

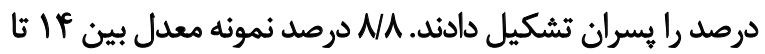

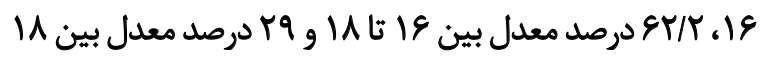

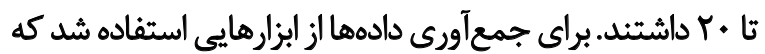

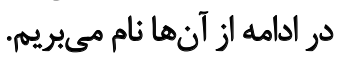

خودكارآمدى تحصيلى: يرسشنامه خودكارآمدى تحصيلى

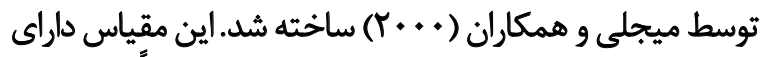

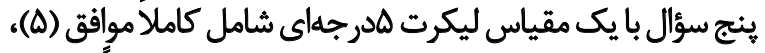

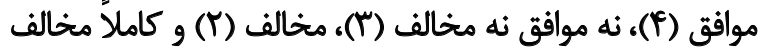

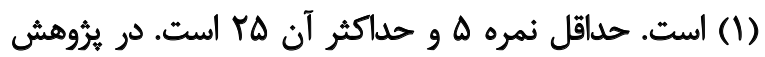

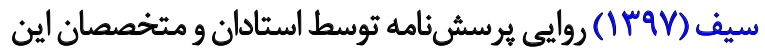

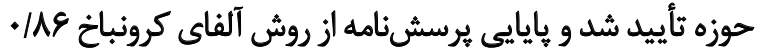

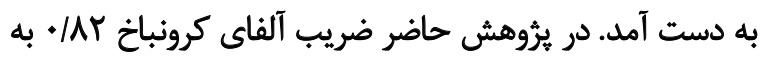
دست آمد.

فرسودگى تحصيلى: يرسشنامه فرسودگى تحصيلى توسط

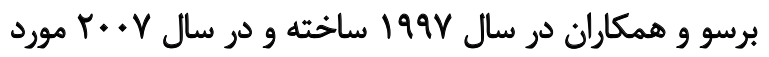

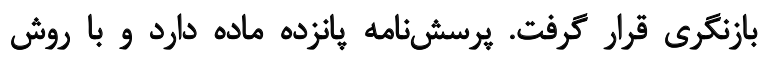

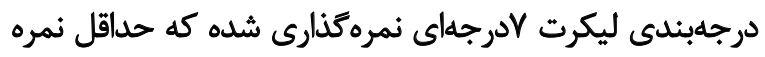

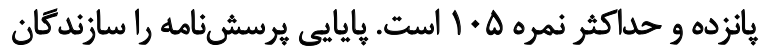

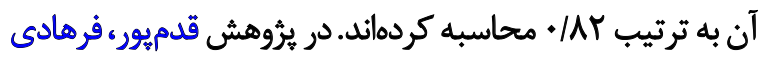

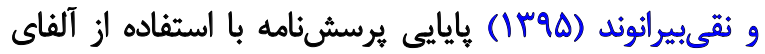

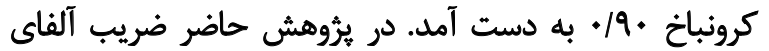

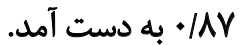

يرسش يرنامه راهبردهاى يادكيرى: زيمرمن و مارتينز يونز در سال

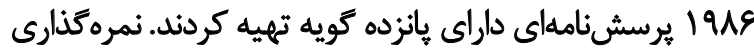

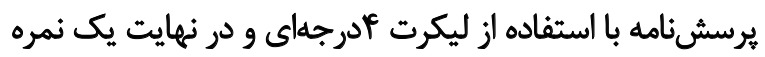

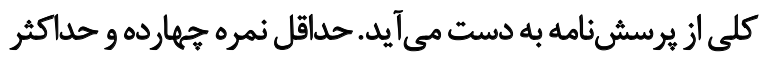

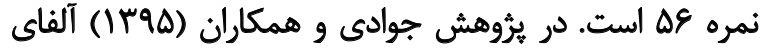

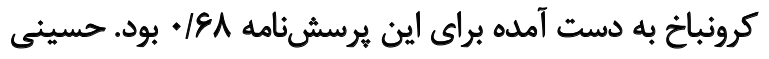

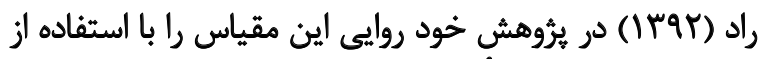

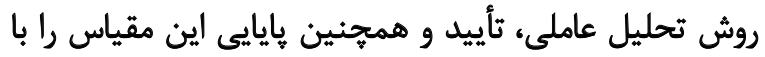

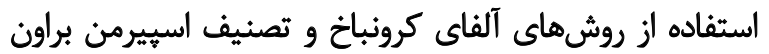

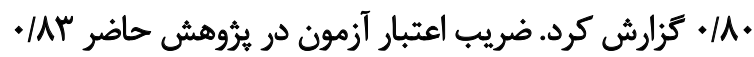

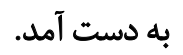

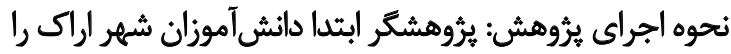

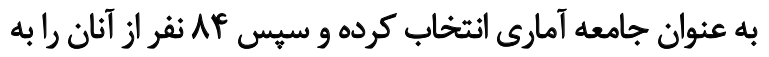

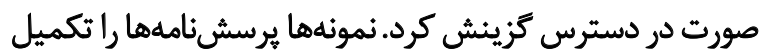

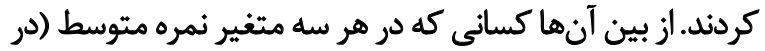

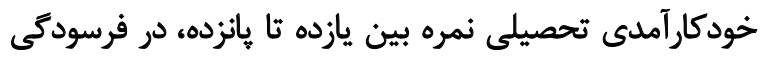

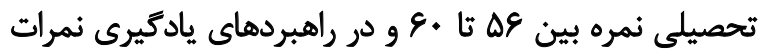




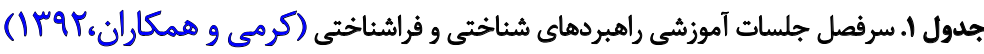

$$
\text { م:تمواى جلسه }
$$

اهداث جلسه

آشننايي با اهداف، روش كار و وظايف افراد

جلسه

معرفي اقراد و مدرس دوره، آشنايي با مفهوم يادئيرى انواع حافظله و ساختار آن و علل فراموشى

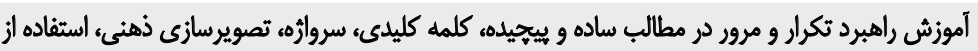
واسطةها و روش مكانها وانها

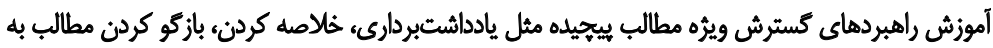
زبان خود

شهح و تقسير و تحليل روابط، استفاده از اطلاعات آموختهشده براي حل مسائل، قياس كرى

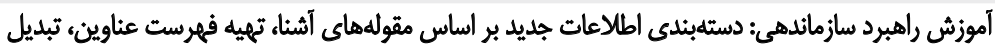

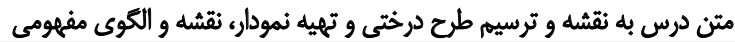

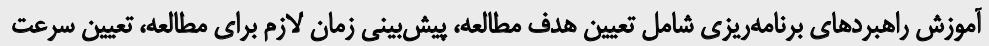

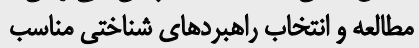

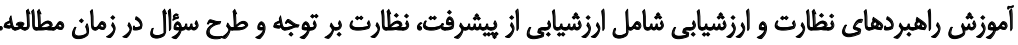

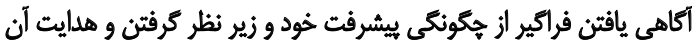

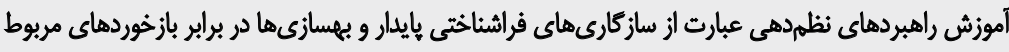

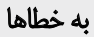

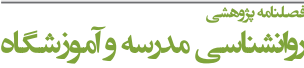

و آموزش روانشناسى مثبتنكر، حداقل بر روى يكى از متغيرهاى

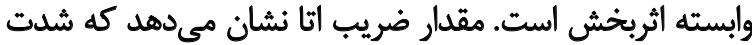

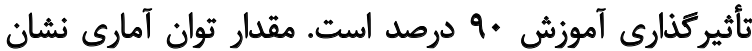

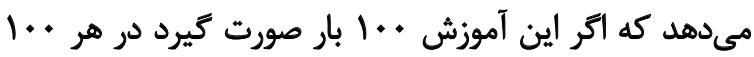

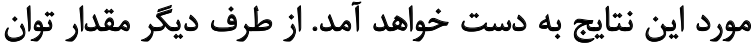
آمارى يك، كفايت حجم نمونه نيز است

نتايج جدول شماره ه هنان مى دهد كه مقدار F محاسبهشده

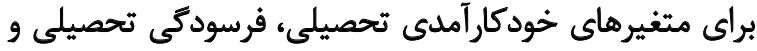
راهبردهاى يادگيرى (در همه متغيرها) معنادار است. به عبارتى فئى فيلى

نشان داد كه مقدار F محاسبهشده براى متغير خودكارآمدى

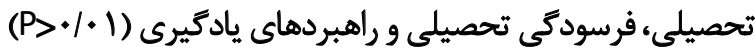

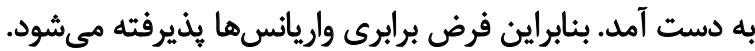

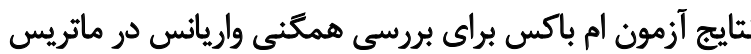

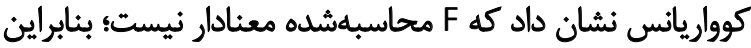

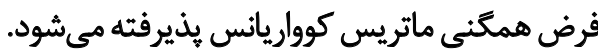
نتايج جدول شماره f نشان مي دهد كه مقدار F محاسبهشده

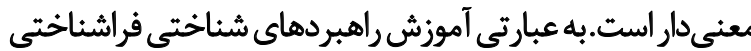

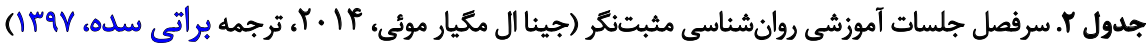

\begin{tabular}{|c|c|c|}
\hline اهداف وخالاصه جلسه & موضوع & 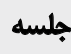 \\
\hline أشنايى و معرفى رهبر و اعضا به يكديكر، بيان اهداف و قواعد كروه، احترام متقابل، شركت منظم و نظاير & آشناييى و همارفه، انجام ارزيابيىهاى اوليه & اول \\
\hline تشويق اعضا به بحث در رابطه با يادئي و يرداختن به هارجوبدهى مجلد & بررسى نوع جهانبينى اعضا نسبت به دنيا و وضعيث & دوم \\
\hline 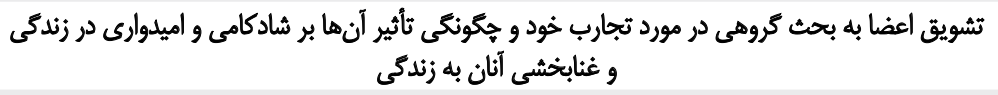 & آشنايى با نقش مثثبتنكرى و اميل و يرداختن & سوم \\
\hline 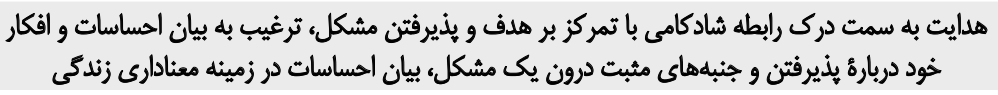 & 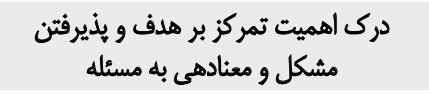 & جهارم \\
\hline 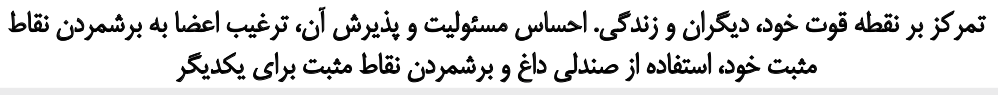 & تمركز بر نقطه قوت خود، ديكران و زندگى & ينجم \\
\hline 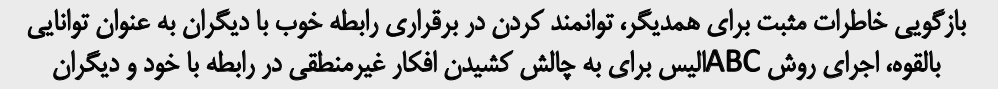 & 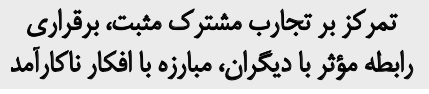 & ششم \\
\hline 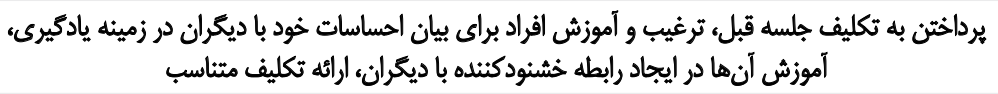 & توانهند كردن اعضا در بيان احساسات ولذت بكديكر & هنهم \\
\hline 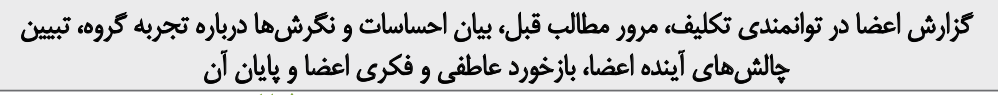 & 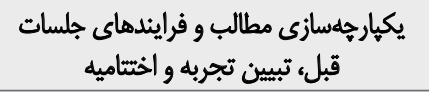 & هشتم \\
\hline
\end{tabular}

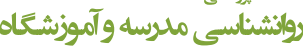




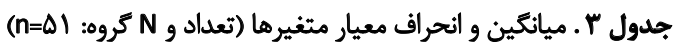

\begin{tabular}{|c|c|c|c|}
\hline \multicolumn{2}{|c|}{ ميانكيّين土 انحراف معيار } & \multirow{2}{*}{ كروه } & \multirow{2}{*}{ متثير } \\
\hline جسأزمون & بيش آزمون & & \\
\hline$\left|r / \kappa^{\prime}+ \pm r / \varepsilon\right|$ & $\mid W /+\varepsilon \pm T / R+$ & 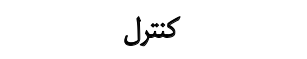 & \multirow{3}{*}{ خودكارآهدى تحصيلى } \\
\hline$r r / r r \pm 1 / v q$ & $\mid$ Weg $\pm / 9 \Delta$ & روانشناسي هثبتنكر & \\
\hline$\Pi \pi / r+ \pm 1 / \% \Delta$ & $1 r / \Delta P \pm 1 / 9 T$ & راهبردهاي شناختى فراشناختى & \\
\hline$\varepsilon \cdot \mid r e \pm 11 / \cdot \varepsilon$ & $\Delta V / V r \pm q / r$ & 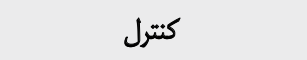 & \multirow{3}{*}{ فرسودكى تحصيلى } \\
\hline$\Pi \pi / M^{\prime \prime} \pm \Delta / u$ & $\Delta N M T \pm \Delta /+\Delta$ & روانشناسى مثبتنكر & \\
\hline re/r. $\pm \varepsilon / 9 q$ & $\Delta N E \cdot \pm 1 \cdot / \Delta E$ & راهبردهاى شناختى فراشناختى & \\
\hline$M r / \Delta r \pm r / \wedge q$ & $M \Psi /+\varepsilon \pm \Delta / T$ & كنترل ل & \multirow{4}{*}{ راهبردهاى يادكيرى } \\
\hline$T V / K \& \pm \Delta / R T^{\prime}$ & $r r / M+ \pm \Delta / \cdot r$ & خوددلسوزى & \\
\hline$r v / r \cdot \pm r / m e$ & $M H / . g \pm M / g T$ & روان شئاسى مثبتنكر & \\
\hline$P V / P+ \pm \Delta / P \varepsilon$ & $\pi \pm t / r q$ & راهبردهاي شناختى فراشناختى & \\
\hline
\end{tabular}

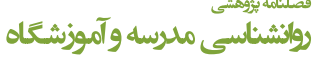

فراشناختى و روانشناسى مثبتنكر بر خودكارآمدى تحصيلى

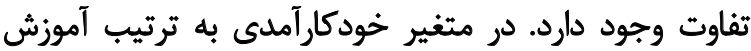

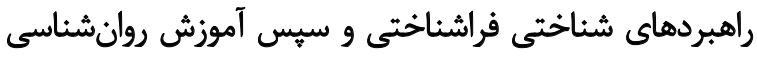

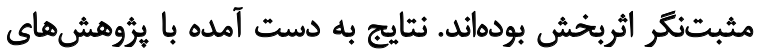

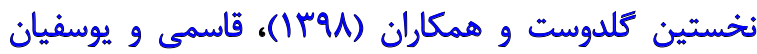

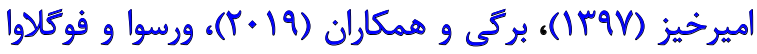

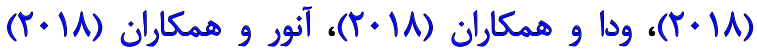

$$
\text { همسوست. }
$$

در تبيين اين نتايج مىتوان كفت عوامل شخصى مانند

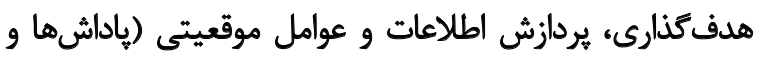

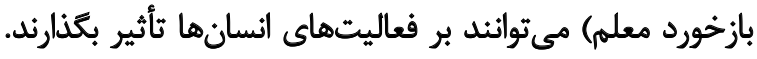

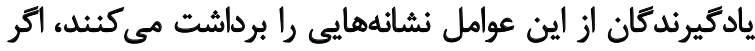

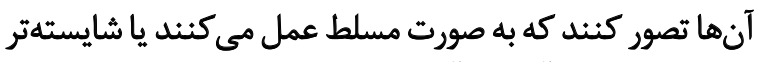

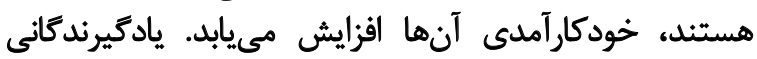

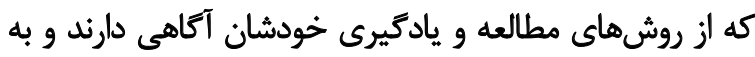

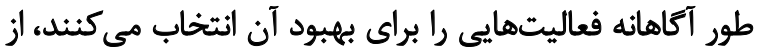

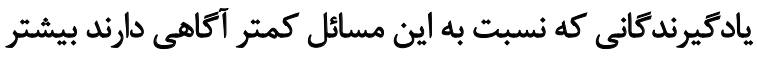

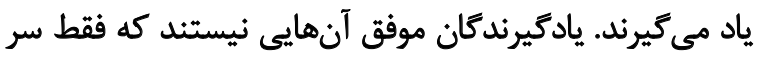

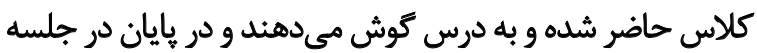

آموزشها تأثير معنى دارى داشته است. مقدار ضريب اتا نشان

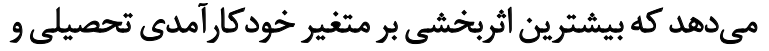

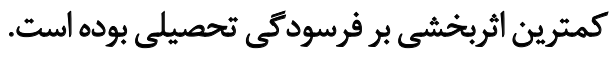
نتايج جدول شماره 9 نشان مي دهد كه در همه متغير مرهاي

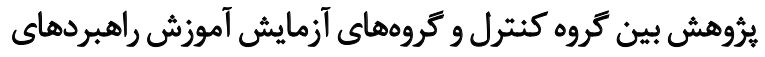

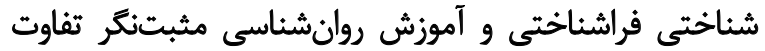

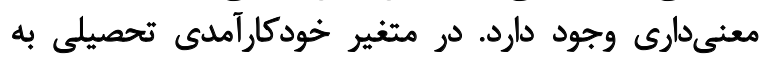

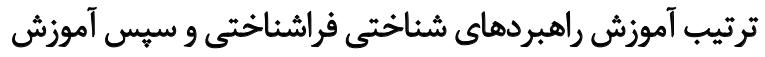

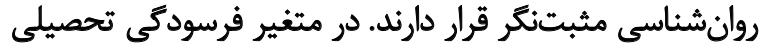

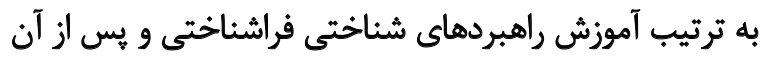

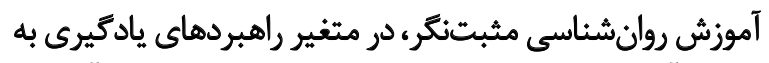

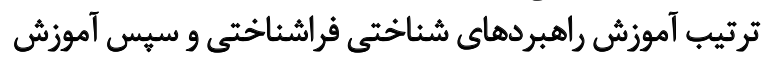
روانشئاسى مثبتنكر اثربخشتر بوده استئ.

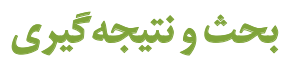

هرئوهش حاضر با هدف سنجش اثربخشى آموزش راهبردهاى

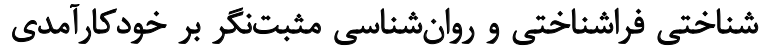

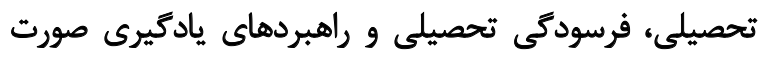
كرفت.نتايج نشان داد بين اثربخشى تحيلى آموزش راهبردير يادهاي شئاختى صورت

جدول P. نتايج آزمون تحليل كوواريانس جندمتغيرى

\begin{tabular}{|c|c|c|c|c|c|c|c|}
\hline توان آمارى & Eta & $\mathbf{P}$ & خط df & فرضيه df & $\mathbf{F}$ & مقدار & آزهون \\
\hline 1 &.$/ 9$. & $.1 . .1$ & $n^{e}$ & 8 & I. T/N & .1 .11 & لامبداي ويلكز \\
\hline
\end{tabular}

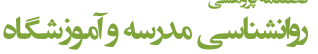


جدول هـ نتايج آزمون تحليل كوواريانس يكراهه در متن تحليل كوواريانس جندمتغيرى

\begin{tabular}{|c|c|c|c|c|c|c|c|}
\hline Eta & $\mathbf{p}$ & $\mathbf{F}$ & MS & df & sS & منيع & مثغير وابسته \\
\hline.$/ 9$. & $.1 . .1$ & $\mid S V / r A$ & $\begin{array}{l}\varphi+\Delta / \mu f \\
r / \Delta \mid\end{array}$ & rq & $\begin{array}{l}11 . / 19 \\
97 / 94\end{array}$ & خين كروهى & خودكارآمدى تحصيلى \\
\hline.$|A|$ & $.1 . .1$ & AT,IT & $\begin{array}{l}\text { M.F/AE } \\
\text { VTNTA }\end{array}$ & $\begin{array}{l}r \\
r q\end{array}$ & $\begin{array}{l}\text { ST.QM } \\
\text { IFASM. }\end{array}$ & خين كروهى & فرسودكى تحصيلى \\
\hline Tar la la & .1 .01 & MFig. & $\begin{array}{l}\text { r. QNEg } \\
\text { NAT }\end{array}$ & r & $\begin{array}{l}\text { PIIV/MT } \\
M r / \% \text {. }\end{array}$ & بين كروهى & راهبردهاى يادكيرى \\
\hline
\end{tabular}

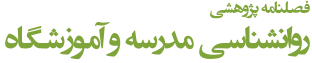

فراشناختى و روانشناسي مثبتنكر بر فرسودگى تحصيلى تفاوت

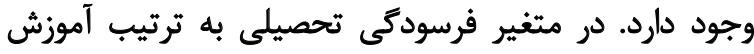

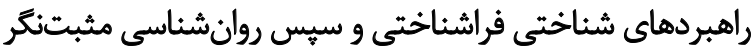

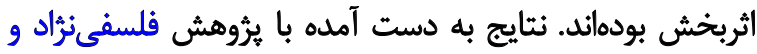

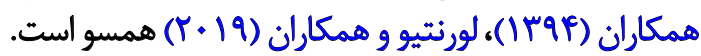

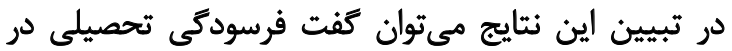

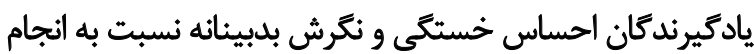

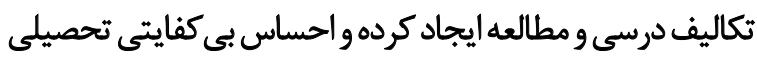

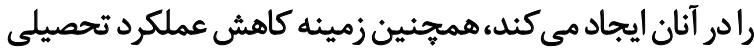

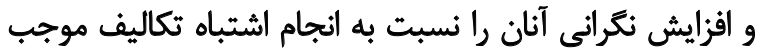

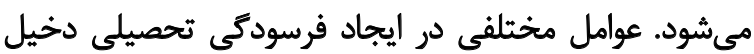

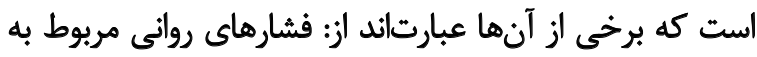

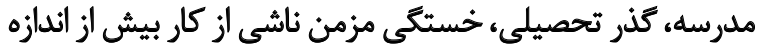

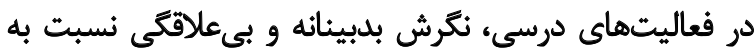

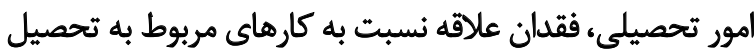

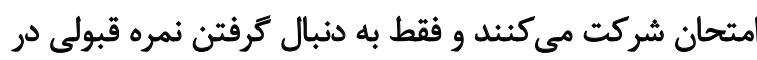

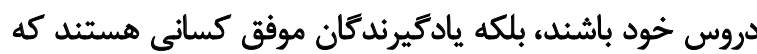

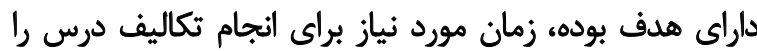

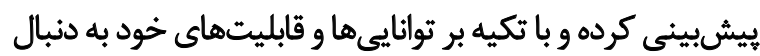

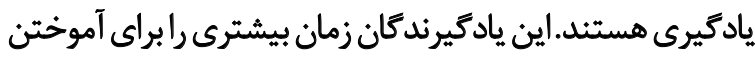

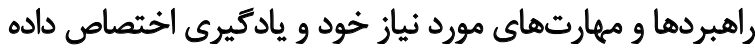

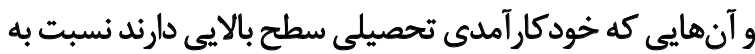

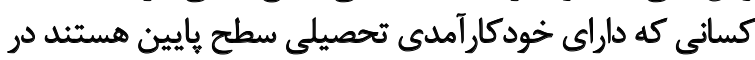

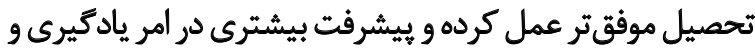

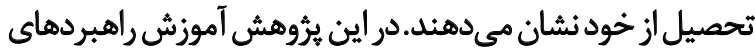

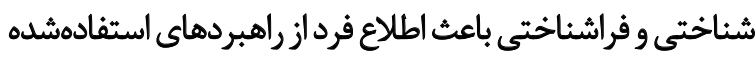

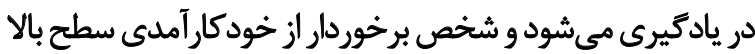

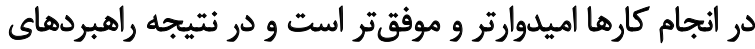

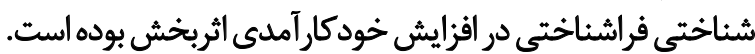
نتايج نشان داد بين اثربخشى آموزش راهبردهاى شناختى

جدول \&. ميانكين تعديلشده و مقايسه زوجي ميانكينها در بسآزمون (آزمون LSD)

\begin{tabular}{|c|c|c|c|c|c|c|}
\hline $\mathbf{P}$ & خطاى معيار & تفاوت ميانكين & كروه تروه & ميانكين士 اتحراف معيار & 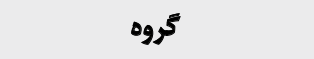 & 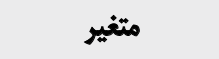 \\
\hline $.1 .+1$ & .194 & $-N \& G$ & كتترل مثبتنكرى & $\mid r / q \cdot \pm \cdot / F \Delta$ & كتترل & \\
\hline.$/ 11$ & .194 & $-\cdot / A V$ & مثبتنكرى راهبردها & TY/KEI./FO & روانشناسى مثبتنكر & خودكارآمدى تحصيلى \\
\hline $.1 .+1$ & .194 & $-9 / \Delta r$ & كتترل راهبردها & $\pi / / \pi \pm \cdot / F a$ & راهبردهاى شناختى فراشناختى & \\
\hline $.1 . .1$ & $r / * 1$ & $r \Delta / A q$ & كتترل مثبتنكرى & $\varepsilon \cdot / \Delta \Psi \pm V / F V$ & كتترل & \\
\hline . & $r / 1$ & $-1 / 79$ & مثبتنكرى راهبردها & $M f / a f \pm V / F V$ & روانشناسى مثبتنكر & فرسودكى تحصيلى \\
\hline $.1 .+1$ & $r / \bullet$ & $r \varphi / \Delta q$ & كنترل راهبردها & $r \Delta / q \uparrow \pm V / F r$ & راهبردهاى شناختى فراشناختى & \\
\hline $.1 .+1$ & $1 / \cdot 9$ & $-V / V q$ & كتترل مثبتنكرى & $F / / \cdot \pm \cdot M$ & كتترل & \\
\hline .1 .01 & $1 / \cdot 9$ & $-\mid Q / T \Delta$ & مثبتنكرى راهبردها & $\mathrm{H} / \mathrm{A} E \pm \cdot \mathrm{W}$ & روانشناسيى مثبتنكر & راهبردهاى يادكيرى \\
\hline $.1 .+1$ & $V / \cdot 9$ & $-r T / . r$ & كنترل راهبردها & $P V / N \pm \cdot M$ & راهبردهاي شناختى فراشناختى & \\
\hline
\end{tabular}

وانشناسي ملرسه وآموزشعاه 
شهر اراك محدود مى شد. همجنين هيرسشنامها تنها ابزار

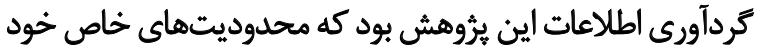

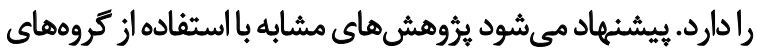

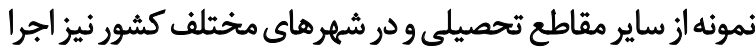

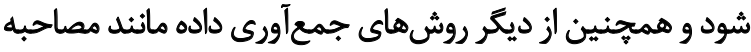

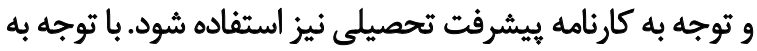

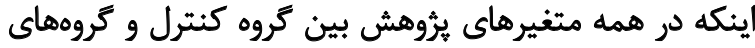

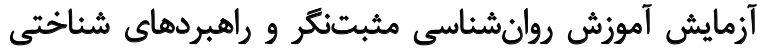

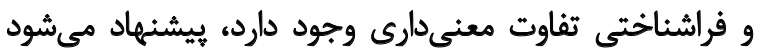
براى افزايش خودكارآمدى تحصيلى و و راهنى

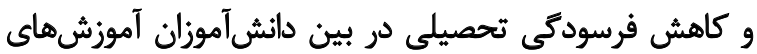

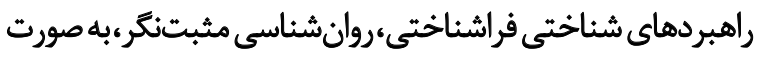

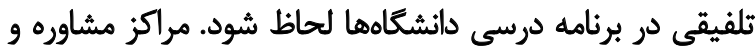

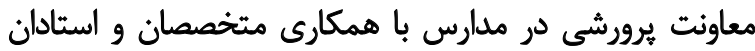

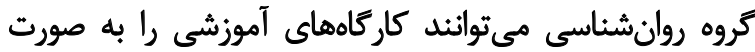

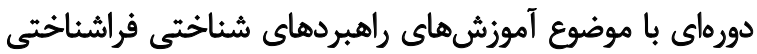

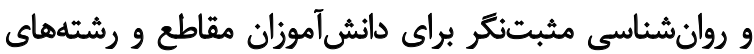

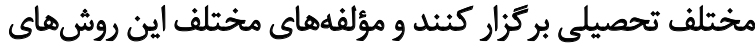
آموزشى را به طور مستقيم به آنان آموزش دهند.

مالاحظات أخلاقي

\section{ييروى از اصول اخلاق ثروهش}

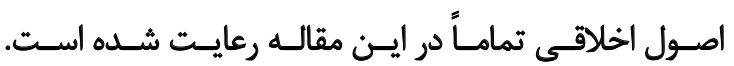

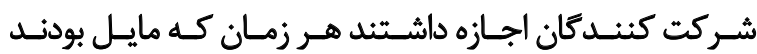

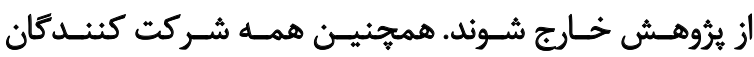

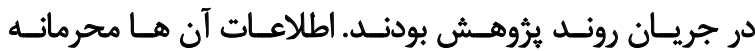

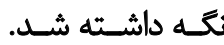

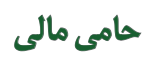

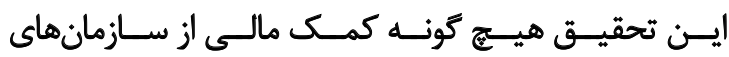

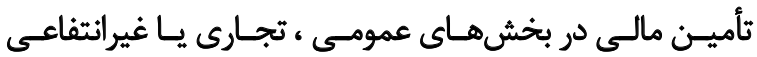
دريافت نكـرد.

مشاركت نويسند مَان

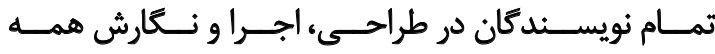

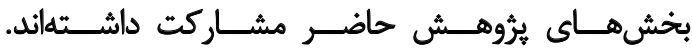

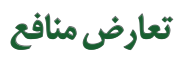

بنابر اظهار نويسندكان اين مقاله تعارض منافع ندارد.
و بيىمعنى ينداشتن آنها، فقدان كارايي مربوط به تحصيل

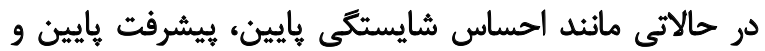

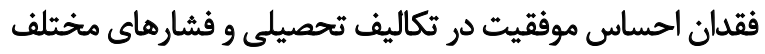

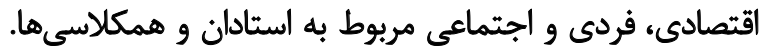

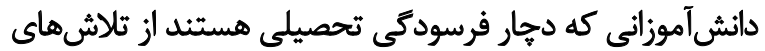

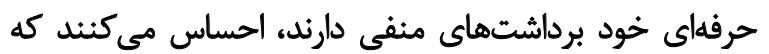

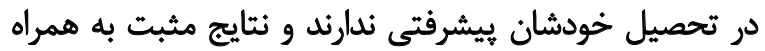

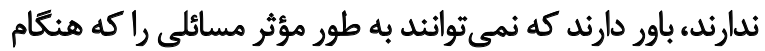

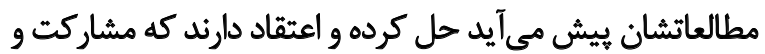

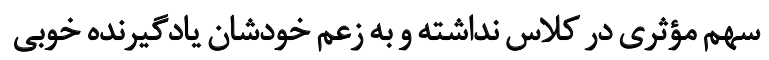

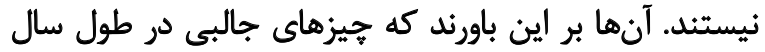

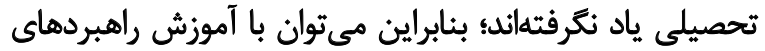

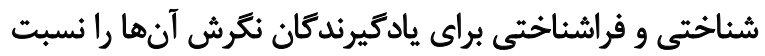

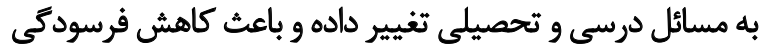

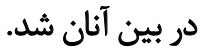

نتايج تحليل كوواريانس نشان داد بين اثربخشى آموزش

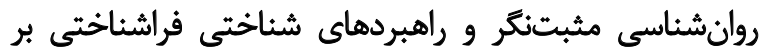

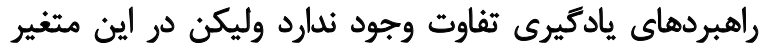

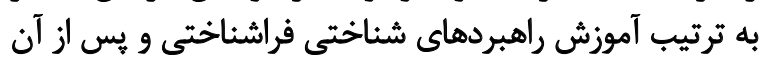

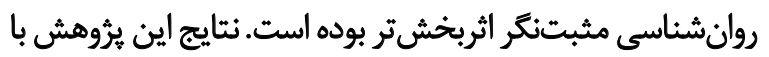

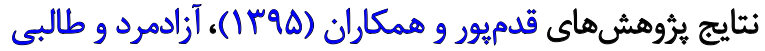

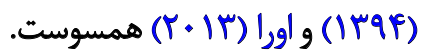

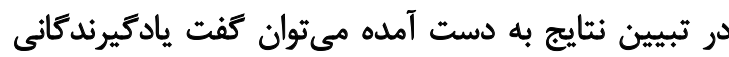

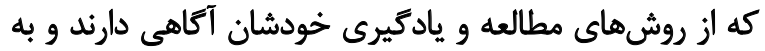

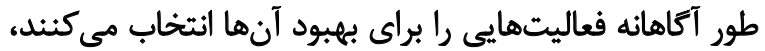

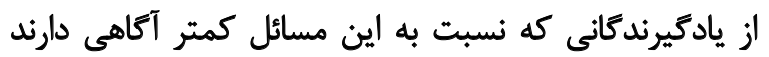

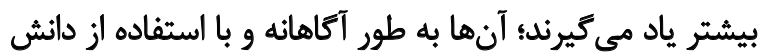

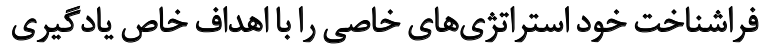

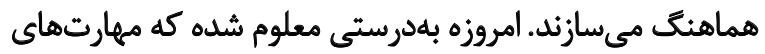

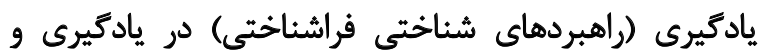

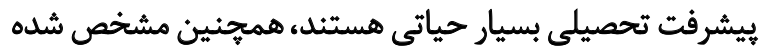

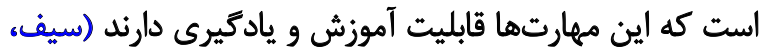

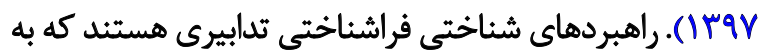

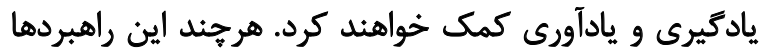

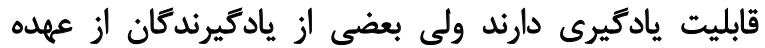

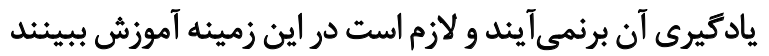

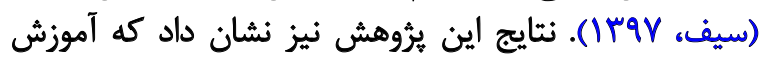

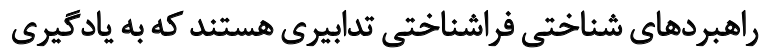

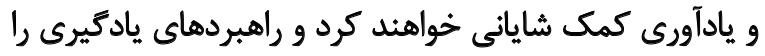

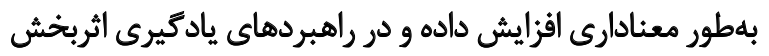
أست.

از محدوديتهاي اين يرؤهش مى توان به جامعه آمارى يُروهش

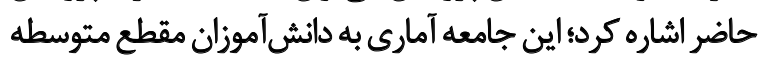




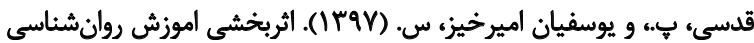

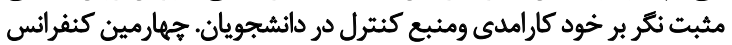

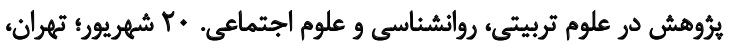
يرديس بين الملل توسعه ايده هزاره.

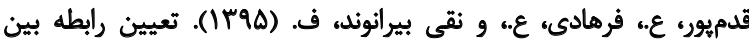

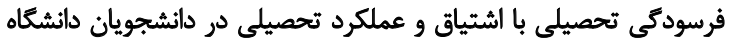

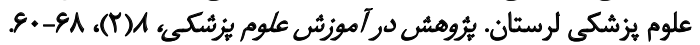

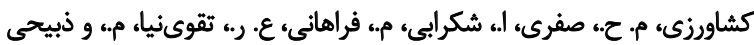

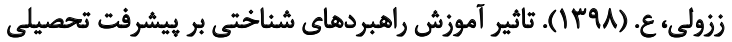

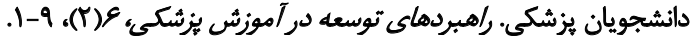

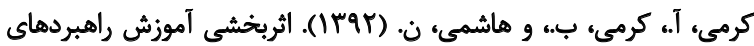

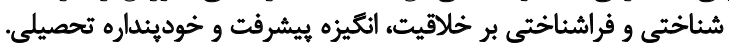

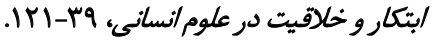

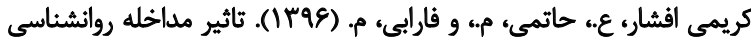

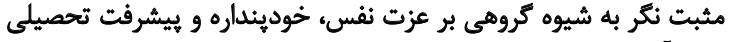

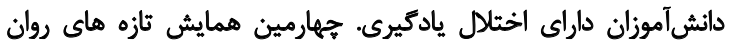

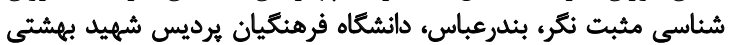

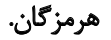

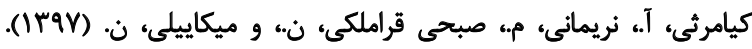

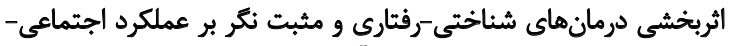

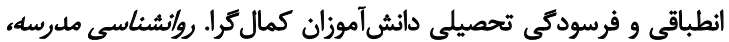
.rF.-rg. (r) $r$

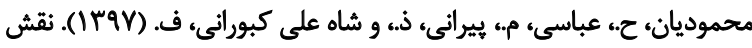

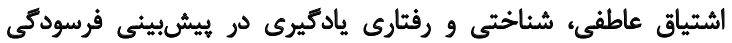

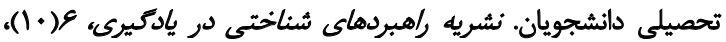

$.19 Y-Y \cdot 9$

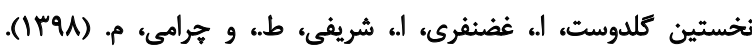

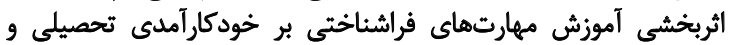

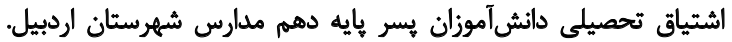

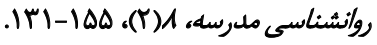

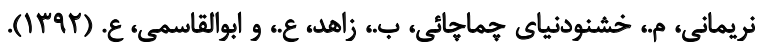

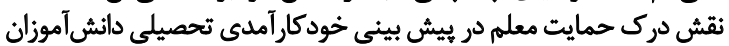

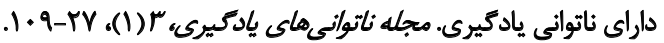

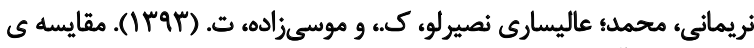

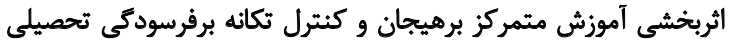

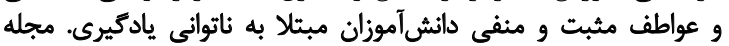

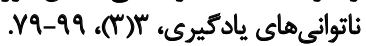

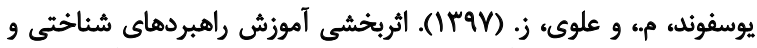

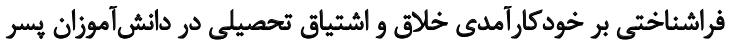

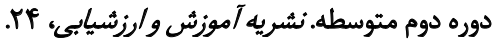

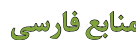

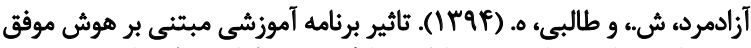

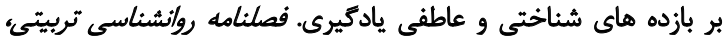

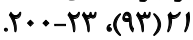

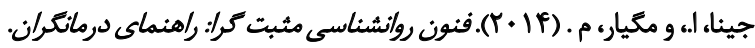

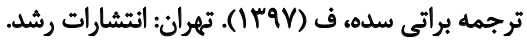

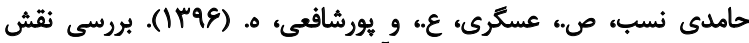

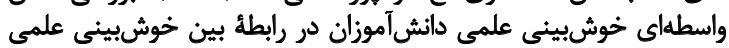

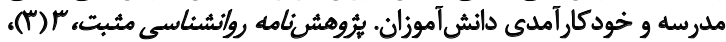
.FY-8.

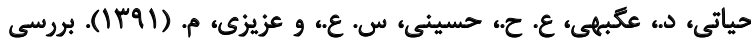

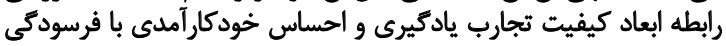

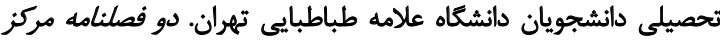

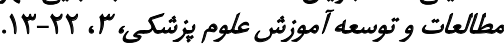

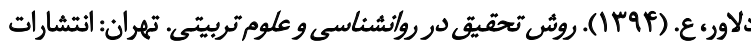
ويرايش.

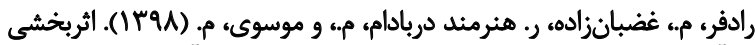

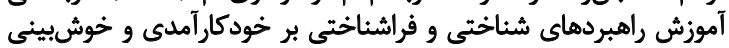

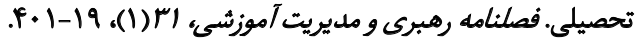

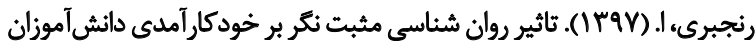

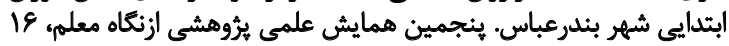

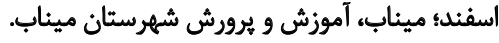

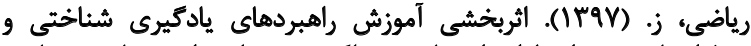

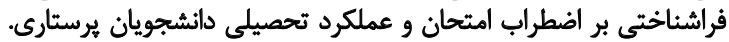

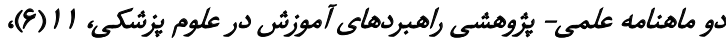
$. P V-\lambda \Delta$

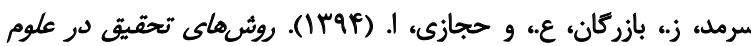

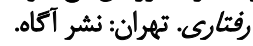

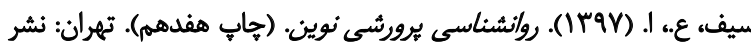
دوران.

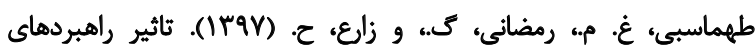

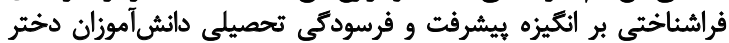

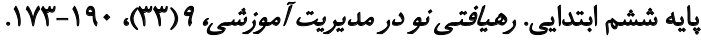

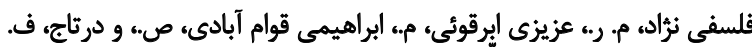

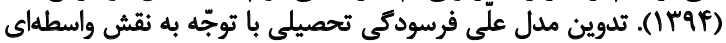

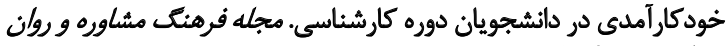

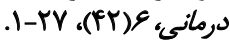

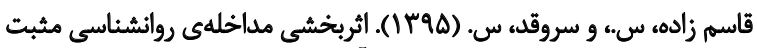

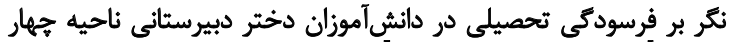

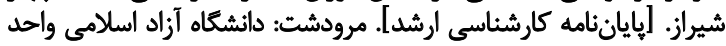

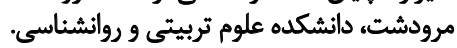

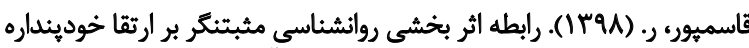

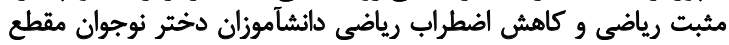

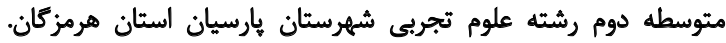

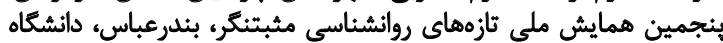
فرهئكيان.

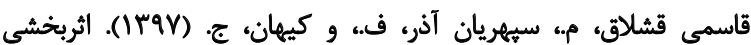

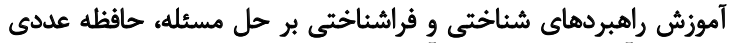

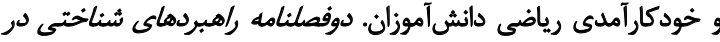

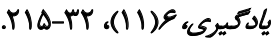




\section{References}

Anuar, N. A. K., Mokhtar, M. I., \& Hasan, H. (2018). Characterizations of Orang Asli (Indigenous) students' learning strategies as related to self-efficacy. Advanced Science Letters, 24(11), 8239-42. [DOI:10.1166/asl.2018.12532]

Al-azzemy, A. F., \& Al-Jamal, D. A. (2019). Evaluating cognitive, metacognitive and social listening comprehension teaching strategies in Kuwaiti classrooms. Heliyon, 5(2), e01264. [DOI:10.1016/j.heliyon.2019.e01264] [PMID] [PMCID]

Aurah, C. M. (2013). The Effects of Self-efficacy Beliefs and Metacognition on Academic Performance. American Journal of Educational Research, 1(8), 334-43. [DOI:10.12691/education-1-8-11]

Azad Mard, Sh., Baqer Kajbaf, M., Faramarzi, S., Talebi, H. (2016). [Comparing the efficiency of successful intelligence-based and conventional education methods on cognitive and affective efficiency of student teachers' learning (Persian)]. Journal of Educational and Scholastic Studies, 2(3), 79-98. http://pma.cfu.ac.ir/article 215. html?lang=en

Bergey, B. W., Parrila, R. K., Laroche, A., \& Deacon, S. H. (2019). Effects of peer-led training on academic self-efficacy, study strategies, and academic performance for first-year university students with and without reading difficulties. Contemporary Educational Psychology, 56, 25-39. [DOI:10.1016/j.cedpsych.2018.11.001]

Breso, E., Salanova, M., \& Schoufeli, B. (2007). In search of the third dimension of Burnout. Applied Psychology, 56(3), 460-72. [DOI:10.1111/j.1464-0597.2007.00290.x]

Brouwers, A., \& Tomic, W. (2000). A longitudinal study of teacher burnout and perceived self-efficacy in classroom management. Teaching and Teacher Education, 16(2), 239-53. [DOI:10.1016/S0742051X(99)00057-8]

Cerezo, R., Fernandez, E., Amieiro, N., Valle, A., Rosario, P., \& Núñez, J. C. (2019). Mediating role of self-efficacy and usefulness between self-regulated learning strategy knowledge and its use. Revista de Psicodidáctica (English ed.), 24(1), 1-8. [DOI:10.1016/j. psicod.2018.08.001]

Chang, S. H., Shu, Y., Wang, C. L., Chen, M. Y., \& Ho, W. S. (2020). Cyber-entrepreneurship as an innovative orientation: Does positive thinking moderate the relationship between cyber-entrepreneurial self-efficacy and cyber-entrepreneurial intentions in Non-IT students? Computers in Human Behavior, 107, 105975. [DOI:10.1016/j. chb.2019.03.039]

Concina, E. (2019). The role of metacognitive skills in music learning and performing: Theoretical features and educational implications. Frontiers in Psychology, 10, 1583. [DOI:10.3389/fpsyg.2019.01583] [PMID] [PMCID]

Delavar, A. (2015). [Research methods in psychology and educational sciences (Persian)]. Tehran: Virayesh Publications. https://www. adinehbook.com/gp/product/9646184022

Eggen, P., \& Kauchak, D. (2001). Educational psychology windows on classrooms. New Jersey: Prentice-Hall, Inc.

Escolano-Pérez, E., Herrero-Nivela, M. L., \& Anguera, M. T. (2019). Preschool metacognitive skill assessment in order to promote educational sensitive response from mixed-methods approach: Complementarity of data analysis. Frontiers in Psychology, 10, 1298. [DOI:10.3389/fpsyg.2019.01298] [PMID] [PMCID]
Dortaj, F., Ebrahimi Ghavamabadi, S., Azizi Abargooi, M., \& Filsafinejad, M. R. (2015). [Developing a causal model of academic burnout among BA students: The mediating role of self-efficacy (Persian)]. Journal of Counseling and Psychotherapy Culture, 6(24), 1-27. http://ensani.ir/file/download/article/20170226045022-9881-92.pdf

Ghadampour, E., Farhadi, A., \& Naghibeiranvand, F. (2016). [The relationship among academic burnout, academic engagement and performance of students of Lorestan University of Medical Sciences (Persian)]. Research in Medical Education, 8(2), 60-8. [DOI:10.18869/ acadpub.rme.8.2.60]

Gasemi Gheshlagh, M., Sepehrianazar, F., \& Keyhan, J. (2018). [The effectiveness of teaching cognitive and metacognitive strategies on problem solving, Numerical memory and Mathematics selfefficacy in students (Persian)]. Biquarterly Journal of Cognitive Strategies in Learning, 6(11), 215-32. [DOI:10.22084/J.PSYCHOGY.2018.14585.1649]

Ghasempor, R. (2019). [The relationship between the effectiveness of positive psychology on promoting positive mathematical self-concept and reducing mathematical anxiety of adolescent female students in parsian scondary school of experimental sciences in Hormozgan Province (Persian)]. Paper presented at The 5th National Conference on Psychological New Positives, Bandar Abbas, Farhangian University, 17 April 2019. https://civilica.com/doc/871051/

Ghasemzadeh, S., \& Sarvghad, S. (2016). [The effectiveness of positive psychology intervention on academic burnout in female high schoo students in Chahar district of Shiraz (Persian)] [MA. thesis]. Shiraz: Islamic Azad University, Marvdasht Branch.

Ghodsi, P., \& Yousefian Amirkhiz, S. (2018). The effectiveness of positive psychology training on self-efficacy and a source of control in students. Paper presented at $4^{\text {th }}$ Research Conference in Educational Sciences, Psychology and Social Sciences. May 12; Tehran, International Campus for the Development of the Millennium Idea.

Halpenny, D., Cadoo, K., Halpenny, M., Burke, J., \& Torreggiani, W. (2010). The Health Professions Admission Test (HPAT) score and leaving certificate results can independently predict academic performance in medical school: Do we need both tests? Irish Medical Journal. [Published Onlion]. https://www.lenus.ie/bitstream/handle/10147/122654/Article5915.pdf?sequence=1

Hamedinasab, S., Asgari, A., \& Pourshafei, H. (2017). [Investigating the mediating role of students academic optimism on the relationship between school academic optimism and students self-efficacy (Persian)]. Positive Psychology, 3(3), 47-60. [DOI:10.22070/ TLR.2020.2528]

Hayati, D., Ogbahi, A., Hoseini Ahangari, S., \& Azizi Abarghuei, M. (2012). [Investigating the relationships between quality of learning experience's components and self-efficacy on academic burnout among students of Allamme Tabatabaei University of Tehran (Persian)]. Educational Development of Judishapur, 3(3), 18-29. http:// journals.ajums.ac.ir/article 90455 aa718e7d01ea7e025bd9655bcb58f8e8.pdf?lang=en

Jeana.1., \& Magyar, M. (2014). Positive Psychology Techniques: A Therapist's Guide. [F. Barati Sadex, Persian Trans]. Tehran: Roshd Publications. http://www.ravanshena30.com/?p=757

Joseph, S., \& Lindley, A. P. (2006). Positive therapy (A meta-theory for psychological practice). New York: Brunner Rout ledge Press.

Karami, B., Allah Karami, A., \& Hashemi, N. (2013). [Effectiveness of cognitive and metacognitive strategies training on creativity, achievement motive and academic self-concept (Persian)]. Journal of Innovation and Creativity in Humanities, 2(4), 122-39. http://journal.bpj. ir/article_523671_a46cfbf9373ef20ebe6c881a364ff9d5.pdf 
Keshavarzi, M. H., Safari, E., Shakarabi, M., Kangrani Farahani, A. R., Taghavinia, M., Zabihi Zazoly, A. (2019). [The effect of teaching cognitive strategies on the academic achievement of medical students (Persian)]. Development Strategies in Medical Education Quarterly, 6 (2), 1-9. [DOI:10.29252/dsme.6.2.1]

Kiamarsi, A., Narimani, M., Sobhi, N., \& Mikaeili, N. (2018). [The effectiveness of cognitive-behavioral and positive therapies on adaptive-social performance and academic burnout of perfectionist students (Persian)]. Journal of School Psychology, 7(2), 240-60. [DOI:10.22098/JSP.2018.702]

Laurențiu, P., \& Maricuțoiu, C. S. (2019). Evolution of self-efficacy, student engagement and student burnout during a semester. A multilevel structural equation mode ling approach. Learning and Individual Differences, 76, 101785. [DOI:10.1016/j.lindif.2019.101785]

Liu, W., Yangu, P., Xiaoman, L., Lixia,W., \& Weiguo, P. (2017). Active procrastination and creative ideation: The mediating role of creative self-efficacy. Personality and Individual Differences, 119(1), 227-9. [DOI:10.1016/j.paid.2017.07.033]

Midgley, C., Maehr, M.L., Hruda, L.Z., Anderman, E., Anderman, L., \& Freeman, K. E., et al. (2000). Manual for patterns of adaptive learning scales. Ann Arbor: University of Michigan Press.

Nakhostin Goldoost, A., Ghazanfari, A., Sharifi, T., \& Chorami, M. (2019). [The Effect of Metacognitive Skills Training on Academic Self-Efficacy and Academic Eagerness of 10th Grade Male Students in Ardabil (Persian)]. Journal of School Psychology, 8(2), 131-55. http://jsp.uma.ac.ir/article_817.html?lang=en

Narimani, M., Alisari Nasirlou, K., \& , Mosazadeh, T. (2014). [The effect of excitement-focused therapy and impulse control on educational burnout and positive and negative training emotions in students with and without learning disabilities (Persian)]. Journal of Learning Disabilities, 3(3), 79-99. https://www.sid.ir/fa/journal/ViewPaper. aspx? $\mathrm{id}=225090$

Narimani, M., Khoshnoodnia Chomachaei, B., Zahed, A., \& Abolghasemi, A. (2013). [The role of perceived teacher support in predicting academic self-efficacy in students with learning disabilities (Persian)]. Journal of Learning Disabilities, 3(1), 109-27. http://jld.uma. ac.ir/article 146.html

Ormrod, J. E. (2006). Educational psychology: Developing learners. Upper Saddle River, N.J.: Pearson/Merrill Prentice Hall.

Paloș, R., Maricuţoiu, L. P., \& Costea, I. (2019). Relations between academic performance, student engagement and student burnout: A cross-lagged analysis of a two-wave study. Studies in Educational Evaluation, 60, 199-204. [DOI:10.1016/j.stueduc.2019.01.005]

Radfar, M., Ghazbanzadeh, R., Honarmand, M., \& Mosavi, M. (2019). [The effectiveness of teaching cognitive and metacognitive strategies on academic self-efficacy and optimism (Persian)]. Quarterly Journal of Educational Leadership and Management, 13(1), 91-104. http://edu.journals.iau-garmsar.ac.ir/article_665966_902c12e90cb88 b1180e031da0cd5b939.pdf

Ranjbar, E. (2018). The effect of positive psychology on self-efficacy of elementary students in Bandar Abbas. Paper presented at The $5^{\text {th }}$ Scientific Research Conference From The Perspective of Teachers. Minab, Education of Minab City, 7 March 2019. https://civilica.com/ doc/866382/

Riyazi, Z. (2019). [The effectiveness of training cognitive and metacognitive learning strategies on anxiety test and academic performance of nursing students (Persian)]. Education Strategies in Medical Sciences, 11(6), 58-64. http://edcbmj.ir/article-1-1668-en.html
Sarmad, Z., Bazargan, A., \& Hejazi, E. (2015). [Research Methods in behavioral sciences (Persian)]. Tehran: Agah Publications.

Schunk, D. H., \& Zimmerman, B. J. (2006). Competence and contro beliefs: Distinguishing the means and ends. In P. A. Alexander, \& P. H. Winne (Eds.), Handbook of Educational Psychology. Mahwah, NJ: LEA.

Seif, A. (2018). [Modern educational psychology (Persian)]. Tehran Doran Publications.

Seligman, M. E. P. (2011). Authentic Happiness. New York: Free Press.

Tahmasebi, G., Ramezani, G., \& Zare, H. (2018). [Review the impact of metacognitive strategies on achievement motivation and academic burnout of girl students of 6th grade husbanded city (Persian)]. Journal of New Approaches in Educational Administration, 9(33), 173190. http://jedu.miau.ac.ir/article_2971_77f77d58309fc343369994d 0c78546e6.pdf

Teng, F., \& Reynolds, B. L. (2019) . Effects of individual and group metacognitive prompts on EFL reading comprehension and incidental vocabulary learning. Plops One, 14(5), e0215902. [DOI:10.1371/ journal.pone.0215902] [PMID] [PMCID]

Verešová, M., \& Foglová, L. (2018). Academic Self-Efficacy, Approach to Learning and Academic Achievement. [Publish Online] [DOI:10.5772/intechopen.70948] [PMID]

Weda, S., Samad, I., Andi, A. P., \& Siti S. F. (2018). The effects of self-efficacy belief, motivation, and learning strategies on students' academic performance in English in higher education. Asian EFL Journal 20(9.2), 140-168. https://www.researchgate.net/publication/327954392

Wood, A. M., \& Joseph, S. (2010). The absence of positive psychological (eudaemonic) well-being as a risk factor for depression: A ten year cohort study. Journal of Affective disorders, 122(3), 213-7. [DOI:10.1016/j.jad.2009.06.032] [PMID]

Yousef Vand, M., \& Alavi, Z. (2018). [The effectiveness of Cognitive and Metacognitive Strategies (CMST) on creative self and passion for education in secondary school students (Persian)]. Journal of Instruction and Evaluation, 11(42), 143-59. http://ensani.ir/file/download/article/1557658146-10100-97-15.pdf

Zimmerman, B., \& Kitsantas, A. (2005). Homework practices and academic achievement: The mediating role of self-efficacy and perceived responsibility beliefs. Contemporary Educational Psychology, 30, 397-417. [DOI:10.1016/j.cedpsych.2005.05.003] 
This Page Intentionally Left Blank 